\title{
Accuracy and Applicability of the New Exchange Correlation Functionals for Reproduction of the Infrared Spectra of Butyl Acrylate and Butyl Methacrylate Molecules
}

\author{
O. Belaidi, ${ }^{1}$ T. Bouchaour, ${ }^{1}$ and U. Maschke ${ }^{2}$ \\ ${ }^{1}$ Laboratoire de Recherche sur les Macromolécules, Faculté des Sciences, Université Abou Bakr Belkaid, BP 119, \\ 13000 Tlemcen, Algeria \\ ${ }^{2}$ Laboratoire de Chimie Macromoléculaire, CNRS-UMR 8009, Bâtiment C6, Université des Sciences et Technologies de Lille, \\ 59655 Villeneuve d'Ascq Cedex, France
}

Correspondence should be addressed to O. Belaidi; othmanblaidi@gmail.com

Received 13 February 2013; Revised 24 April 2013; Accepted 2 September 2013

Academic Editor: William N. Setzer

Copyright (C) 2013 O. Belaidi et al. This is an open access article distributed under the Creative Commons Attribution License, which permits unrestricted use, distribution, and reproduction in any medium, provided the original work is properly cited.

The butyl acrylate and butyl methacrylate were optimized by seven functionals. All the structures found are local minima and belong to the Cs symmetry. The calculated frequencies are scaled and ranked according to their square errors. The scaling factors of the B972 and B98 functionals fail to reproduce the infrared spectra. The calculated and scaled frequencies with G96LYP, OLYP, and HCTH functionals give acceptable correlations with the experimental spectra. The scaling factors for O3LYP/6-31G(f,p) and $\mathrm{O} 3 \mathrm{LYP} / 6-311+\mathrm{G}(\mathrm{df}, \mathrm{p})$ levels of theory reproduce very well the infrared spectrum of butyl acrylate, and the scaled frequencies at VSXC functional with Pople's double zeta basis sets show the best accuracy in the case of butyl methacrylate.

\section{Introduction}

The density functional theory is one of the most efficient and promising methods of quantum physics and chemistry. It is a theory of electronic structure formulated in terms of electronic density as the basic unknown function instead of the electron wave function. According to the fundamental theorem of Hohenberg and Kohn, the electron density carries all the information that one might need to determine any propriety of the electron system. This methodology is the most used by scientists for predicting the molecular structures and reproducing the experimental data. It is well established from the large amount of the published paper on the calculated molecular properties that the DFT is more accurate and less time consuming than the ab initio methods. Unfortunately, the exchange correlation functional is known as an approximate form, and there is no systematic way to improve its accuracy as in the conventional ab initio methodology. In the last decade, many approximate exchange-correlation functionals have been introduced to the scientific community $[1,2]$. To improve the accuracy of these functionals, one should apply them to medium and large systems. A considerable amount of reports on the vibrational frequency calculations are available in the literature using the earlier exchange correlation functions, namely, BLYP, BP86, B3LYP, and B3PW91 [3-8]. Nonetheless, they fail to correctly predict the experimental vibrational spectra. This is due to the deficiency of the electron-electron interaction considered and the neglect of anharmonicity when calculating the vibrational frequencies, and the experimental data are almost adjusted with the simulated one using the scaling factors [9$11]$.

Our objective in this work is to test how accurate the recently developed exchange correlation functionals reproduce the infrared spectra of medium-sized molecules, namely, butyl acrylate and butyl methacrylate. The functionals used in this work, B972 [12, 13], B98 [14], G96LYP [15], HCTH [16], OLYP [1], O3LYP [2], and VSXC [17], were considered as they were implemented in the Gaussian $03 \mathrm{rev}$. B.01 program [18].

To the best of our knowledge, no one has tested the accuracy of this set of functionals on medium-sized molecules. 
However, there have been some works which made an assessment on the prediction of the infrared spectra. Jaramillo and Scuseria report that the performance of VSXC is comparable to the experimental and B3LYP data [19]. In the vibrational analysis of thiosaccharin and thiosaccharinate anion, Jovanovski et al. [20] found that HCTH and OLYP gave comparable results as the B3LYP and they have been used all together for the assignment of bands. Raissi et al. [21] report the vibrational assignment of trifluoroacetyl acetone using B3LYP, B3PW91, BLYP, BPW91, and G96LYP functionals. They did not see the need for use of scaling factors. The G96LYP gave the smallest wavenumbers within this set of functionals.

\section{Experimental}

Butyl acrylate (BuA) and butyl methacrylate (BuMA) (purity greater than 99\%) were purchased from Sigma-Aldrich, and they were used as they are without further purifications. The infrared spectra of liquid Butyl acrylate and butyl methacrylate were recorded within $1800-700 \mathrm{~cm}^{-1}$ range with a Perkin Elmer FTIR System-2000 model.

\section{Computational}

Molecular geometries were determined by gradient optimizations using the Kohn-Sham density functional theory [22]. Seven functionals with different levels of complexity were investigated: B972, B98, G96LYP, HCTH, OLYP, O3LYP, and VSXC. The OLYP, HCTH, and G96LYP functionals are the generalized gradient approximation (GGA) functionals. The B972 and O3LYP are the hybrid functionals. The VSXC functional is the meta-GGA functionals, which depend on the kinetic energy density term. The B98 functional is hybrid meta-GGA functional incorporating $21.98 \% \mathrm{HF}$ exchange. The O3LYP functionals are derived from Lee-yang-Parr correlation functional, the Handy's OPTX exchange functional which reparameterized the Becke8 8 exchange functional with a flexible formula, and the HF exact exchange [1]. For each one of these functionals, the following four basis sets were applied: two of Pople's split valence basis sets 6-31G(d,p) and $6-311+(\mathrm{df}, \mathrm{p})[23-25]$ and two flexible polarized $(\mathrm{p})$ and correlation consistent (cc) valence basis set [26] (cc-p VDZ, AUG-cc-p VDZ). AUG denotes augmentation by diffuse functions. This selection represents a good deal of commonly used basis sets. The butyl acrylate and butyl methacrylate molecules were first optimized at a given methodology, and then harmonic vibrational normal modes were calculated. The obtained frequencies were scaled using the scaling factors proposed by Tantirungrotechai et al. [11]. The scaled frequencies were compared with the experimental ones in the finger print region $1800-700 \mathrm{~cm}^{-1}$. The scaling factors $\lambda$ for vibrational frequencies were evaluated by minimizing the square error between the calculated and the experimental values:

$$
\Delta_{\mathrm{vib}}=\sum_{i}^{\mathrm{all}}\left(\lambda \omega_{i}-\widetilde{v}_{i}\right)^{2}
$$

where $\omega_{i}$ and $\widetilde{v}_{i}$ are the $i$ th calculated harmonic and the $i$ th experimental fundamental frequencies in $\mathrm{cm}^{-1}$, respectively. The used scaling factors are represented in Table 1.

All the calculations were performed by using the Gaussian 03 rev. B01 program running on PCs running the windows operating system [18].

The Figure 1 shows clearly that the scaling factors give more accurate spectra than the unscaled ones. The difference between the scaled and unscaled frequencies rises up to $35 \mathrm{~cm}^{-1}$ for high frequencies.

\section{Results and Discussion}

All the optimizations lead to the Cs symmetry of the butyl acrylate and butyl methacrylate molecules. For molecular structure and atom numbering, see Figure 2. It is too knotty to compare all the observed and calculated frequencies for each spectrum. We based this confrontation on the nine wellknown and prominent bands in the experimental spectrum of butyl acrylate and eight bands for butyl methacrylate. The observed bands values and assignments are shown in Table 2.

The first point that should be discussed is the influence of the basis sets used during the simulation for each level of theory. We notice a bit changes in each spectrum but this would not affect the general quality of the functionals used. The differences in the frequency for a given band do not exceed $30 \mathrm{~cm}^{-1}$.

When exploring the different Figures 3, 4, 5, 6, 7, 8, 9, $10,11,12,13,14,15$, and 16 , we notice that the accuracy of the simulated spectra for the mentioned functionals would be classified into three classes. So we calculate the percentage of the sum of square errors of each level of theory. The hundred percent is the highest value of the sum of square errors found within the 28 spectra calculated for each molecule. This is explicitly shown in

$$
\% \Delta_{\mathrm{vib}_{i}}=\frac{\Delta_{\mathrm{vib}_{i}} * 100}{\Delta_{\mathrm{vib}_{\max }}}
$$

where $\% \Delta_{\mathrm{vib}_{i}}$ is the percentage of the sum of square errors of each functional/basis set level of theory, $\Delta_{\mathrm{vib}_{i}}$ is the sum of square errors of a given functional/basis set system, and $\Delta_{\text {vib }_{\max }}$ is the maximum value of $\Delta_{\mathrm{vib}_{i}}$ found through the 28 systems (functionals/basis set). Then the ranking is made on the basis of $\% \Delta_{\mathrm{vib}_{i}}$ values. When $\% \Delta_{\mathrm{vib}_{i}}<1 \%$, we have good correlated spectra, $1 \% \leq \% \Delta_{\text {vib }_{i}} \leq 4 \%$ acceptable spectra, and for $\% \Delta_{\mathrm{vib}_{i}}>4 \%$, we have bad correlate spectra.

4.1. Bad Correlated Spectra. Figures 3 to 6 represent a comparison between the experimental infrared spectrum and the simulated spectra for butyl acrylate and butyl methacrylate, respectively. In these figures, the $\mathrm{B} 972$ and B98 functionals are used. The spectra at these levels of theory result into a very bad reproduction of the infrared experimental spectrum of the title molecules. The calculated and scaled frequencies for both functionals less estimate the experimental bands. This deficiency overcomes the barrier of $200 \mathrm{~cm}^{-1}$. The calculated $\% \Delta_{\mathrm{vib}_{i}}$ is superior to $80 \%$ for butyl acrylate and $75 \%$ for butyl 
TABLE 1: The vibrational frequency scaling factors evaluated for different exchange correlation functionals and basis sets.

\begin{tabular}{lcccccrr}
\hline & B972 & B98 & G96LYP & HCTH & OLYP & O3YP & VSXC \\
\hline 6-31G(d,p) & 0.9532 & 0.9625 & 0.9936 & 0.9713 & 0.9788 & 0.9615 & 0.9686 \\
6-31+G(df,p) & 0.9593 & 0.9679 & 0.9978 & 0.9772 & 0.9849 & 0.9678 & 0.9740 \\
cc-pVDZ & 0.9618 & 0.9710 & 1.0013 & 0.9791 & 0.9875 & 0.9696 \\
Aug-cc-pVDZ & 0.9606 & 0.9707 & 1.0029 & 0.9790 & 0.9873 & 0.9701 & 0.9770 \\
\hline
\end{tabular}

TABLE 2: Observed bands for butyl acrylate and butyl methacrylate with their assignments.

\begin{tabular}{|c|c|c|c|c|}
\hline & $\begin{array}{c}\text { Butyl acrylate } \\
\text { Observed band }\left(\mathrm{cm}^{-1}\right)\end{array}$ & Assignment & $\begin{array}{c}\text { Butyl methacrylate } \\
\text { Observed band }\left(\mathrm{cm}^{-1}\right)\end{array}$ & Assignment \\
\hline$v_{1}$ & 812 & $\mathrm{C}_{1} \mathrm{H}_{2}$ out of plane bending & 812 & $\mathrm{C}_{1} \mathrm{H}_{2}$ out of plane bending \\
\hline$v_{2}$ & 984 & $\mathrm{C}_{1} \mathrm{H}_{5}$ out of plane bending & 937 & $\mathrm{C}_{1} \mathrm{H}_{2}$ in plane bending \\
\hline$v_{3}$ & 1066 & CCC deformation & 1019 & CCC deformation \\
\hline$v_{4}$ & 1188 & OC stretching & 1161 & OC stretching \\
\hline$\nu_{5}$ & 1279 & $\mathrm{C}_{1} \mathrm{H}_{2}$ twisting & 1319 & $\mathrm{C}_{1} \mathrm{H}_{2}$ twisting \\
\hline$v_{6}$ & 1408 & OCC deformation & 1457 & $\mathrm{CH}_{2}$ twisting \\
\hline$v_{7}$ & 1464 & $\mathrm{CH}_{2}$ twisting & 1638 & $\mathrm{C}=\mathrm{C}$ stretching \\
\hline$\nu_{8}$ & 1623 & $\mathrm{C}=\mathrm{C}$ stretching & 1717 & $\mathrm{C}=\mathrm{O}$ stretching \\
\hline$v_{9}$ & 1727 & $\mathrm{C}=\mathrm{O}$ stretching & & \\
\hline
\end{tabular}
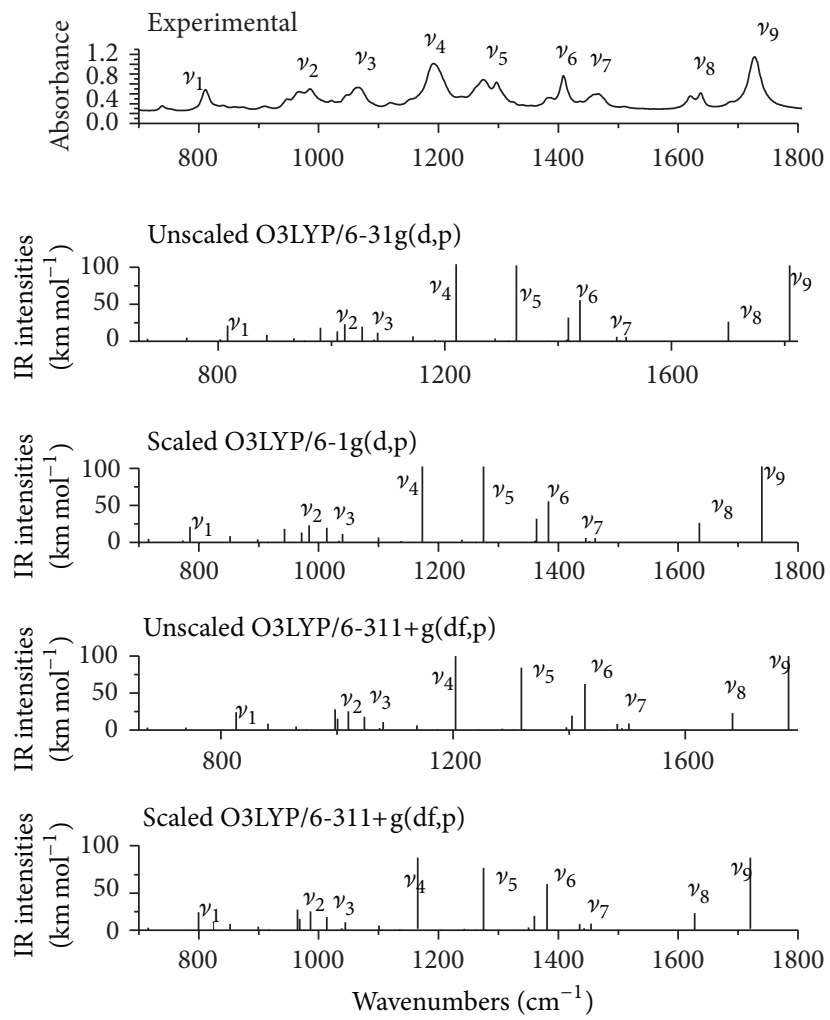

FIGURE 1: The difference between the scaled and unscaled frequencies calculated at O3LYP/6-31g(d,p) and O3LYP/6-311+g(df,p) level of theory for butyl acrylate molecule. The scaling factors used are 0.9615 and 0.9678 . 


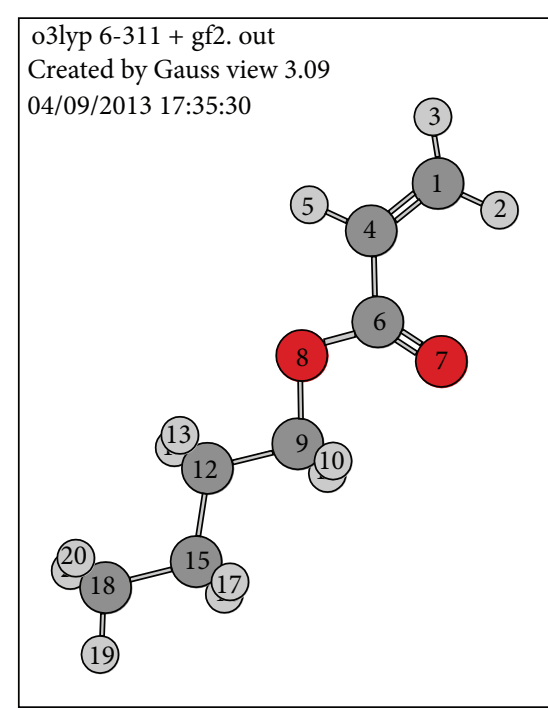

(a)

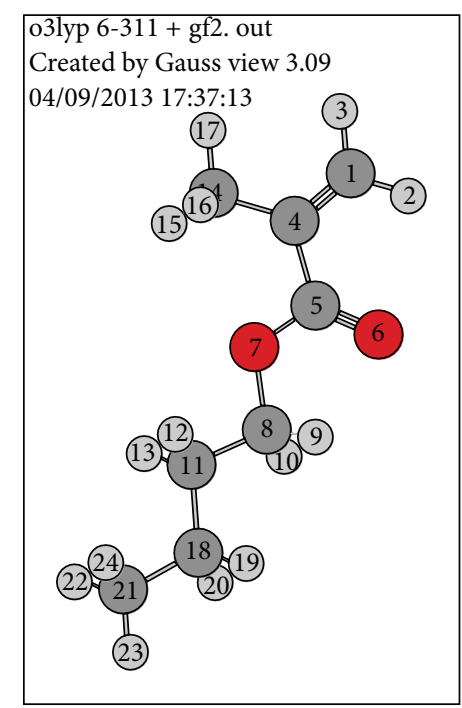

(b)

FIGURE 2: Chemical structure of butyl acrylate and butyl methacrylate molecules.
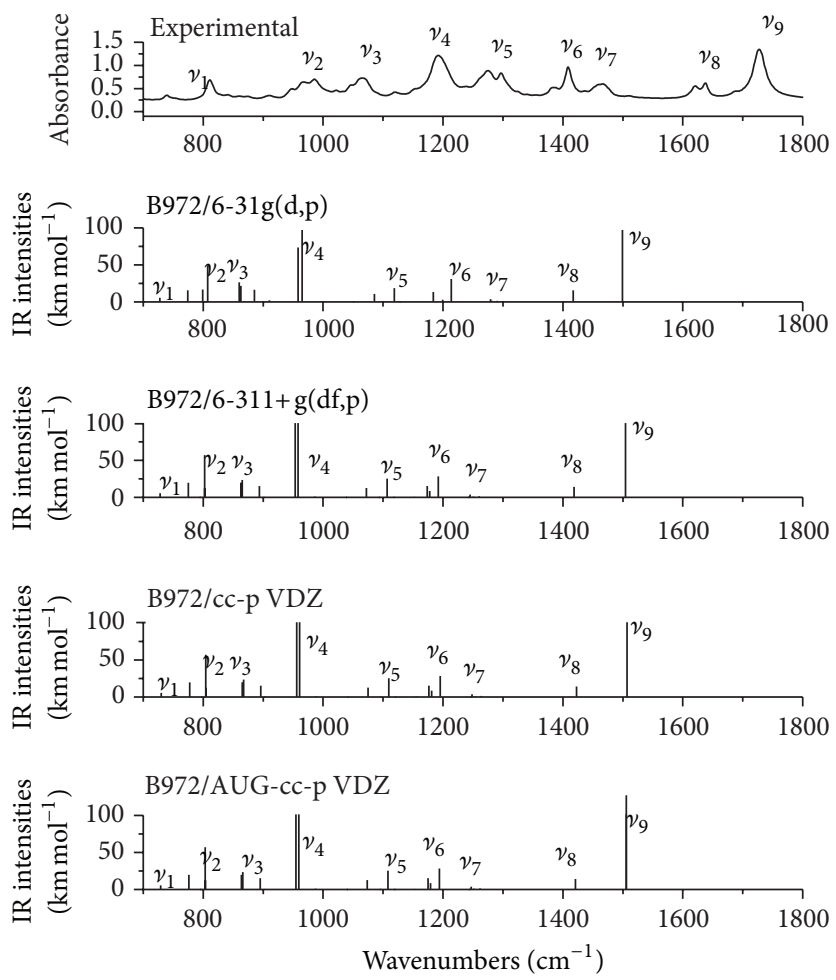

FIGURE 3: Comparison between the experimental infrared spectrum of butyl acrylate and the simulated spectra using different basis sets at the B972 functional.

methacrylate. The B98 functional gives the maximum errors for both molecules.

4.2. Acceptable Correlated Spectra. All the calculated frequencies at the G96LYP level of theory give a less estimation of the experimental ones (see Figures 6 and 7). The 6$31 G(d, p)$ basis set is the best choice for this functional for both molecules. The difference between the experimental and the calculated frequencies does not exceed $30 \mathrm{~cm}^{-1}$. However, all the basis sets used fail to give an acceptable estimation for the $\nu_{3}$ band in the butyl acrylate spectrum. The calculated $\% \Delta_{\text {vib }}$ for $\mathrm{BuA}$ are greater than $1.81 \%$, and less than $2.18 \%$ for BuMA.

Figures from 9 to 11 show the comparison between the infrared and the simulated spectra for BuA using $\mathrm{HCTH}$, 

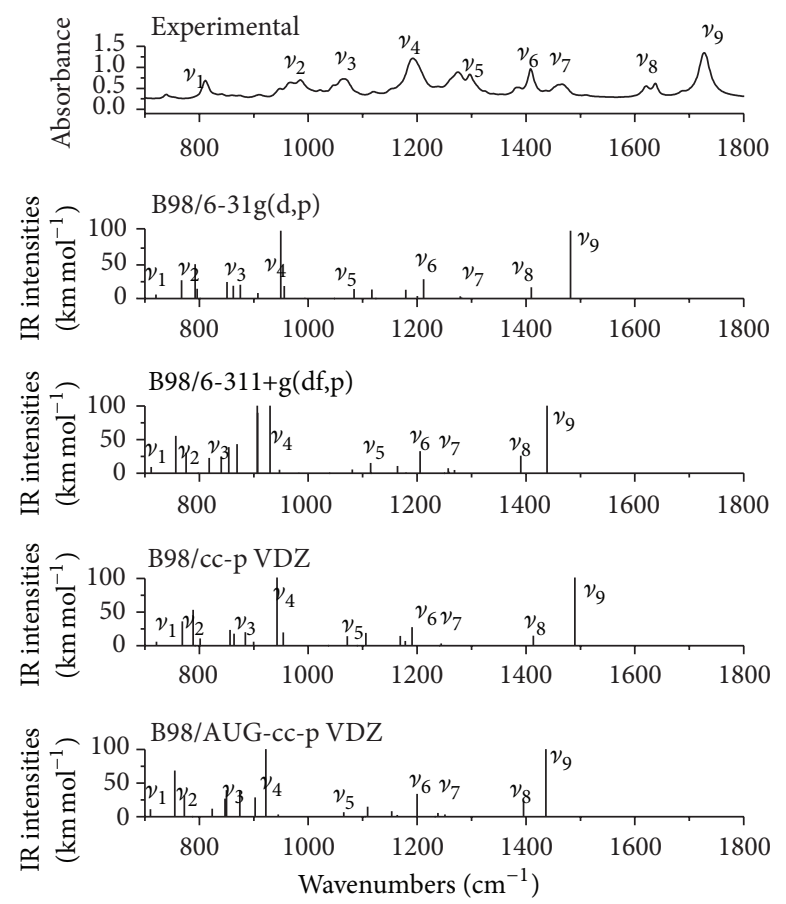

FIGURE 4: Comparison between the experimental infrared spectrum of butyl acrylate and the simulated spectra using different basis sets at the $\mathrm{B} 98$ functional.
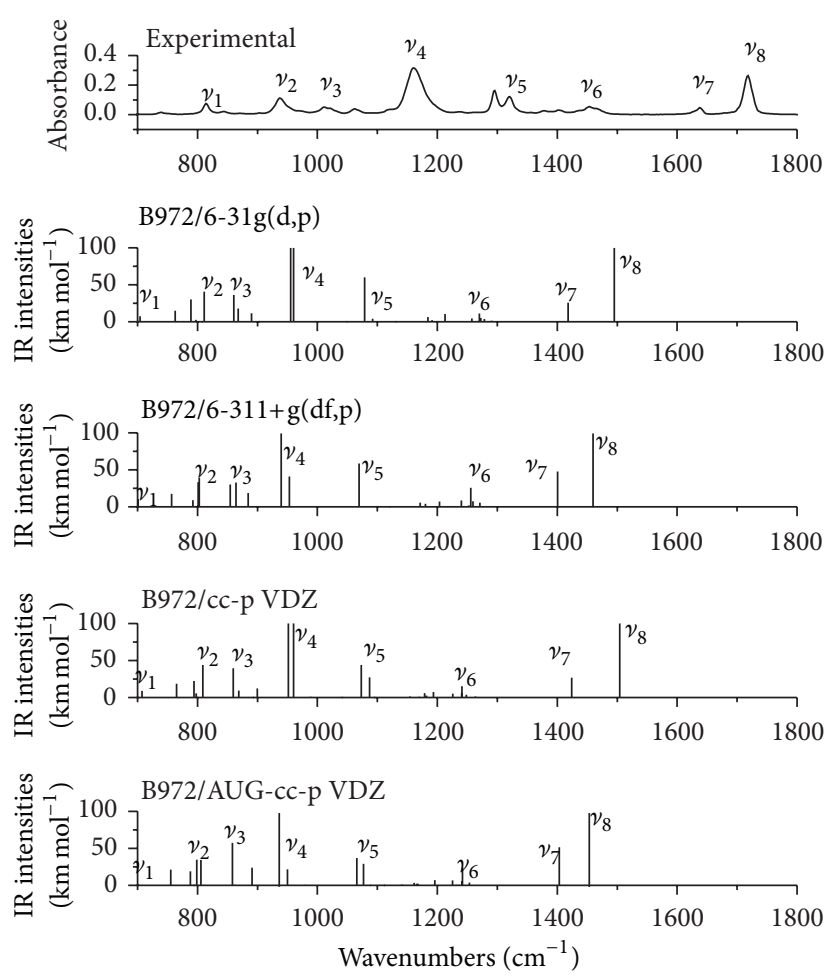

FIGURE 5: Comparison between the experimental infrared spectrum of butyl methacrylate and the simulated spectra using different basis sets at the B972 functional. 

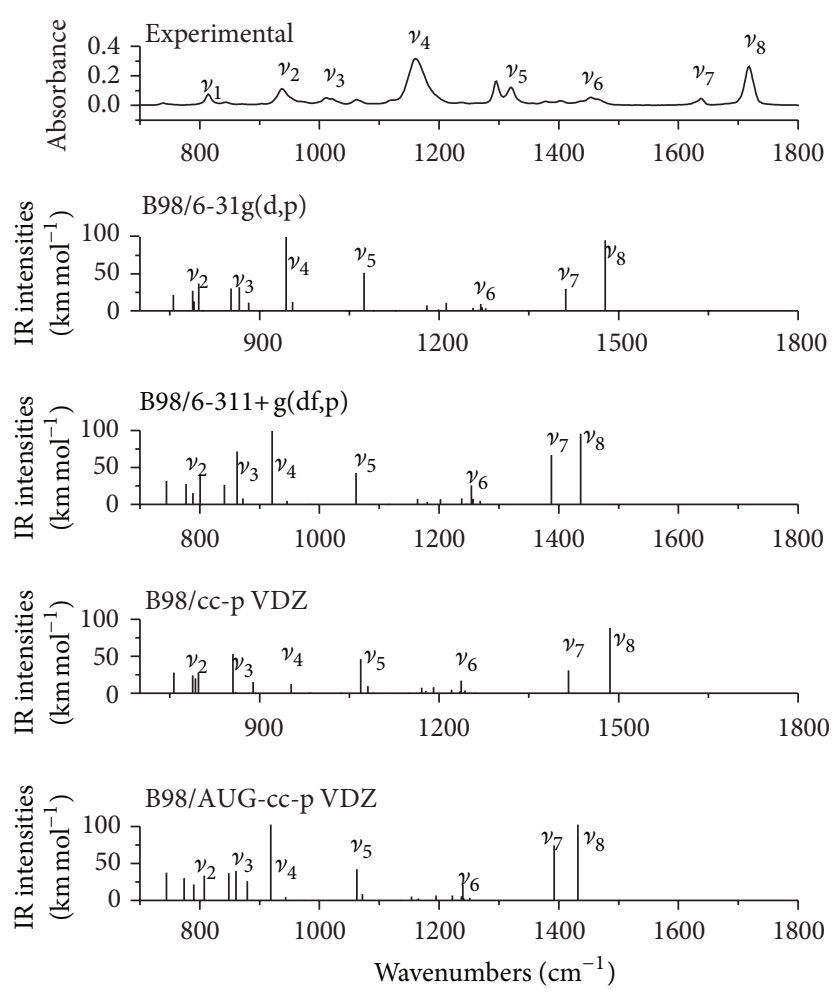

FIGURE 6: Comparison between the experimental infrared spectrum of butyl methacrylate and the simulated spectra using different basis sets at the $\mathrm{B} 98$ functional.
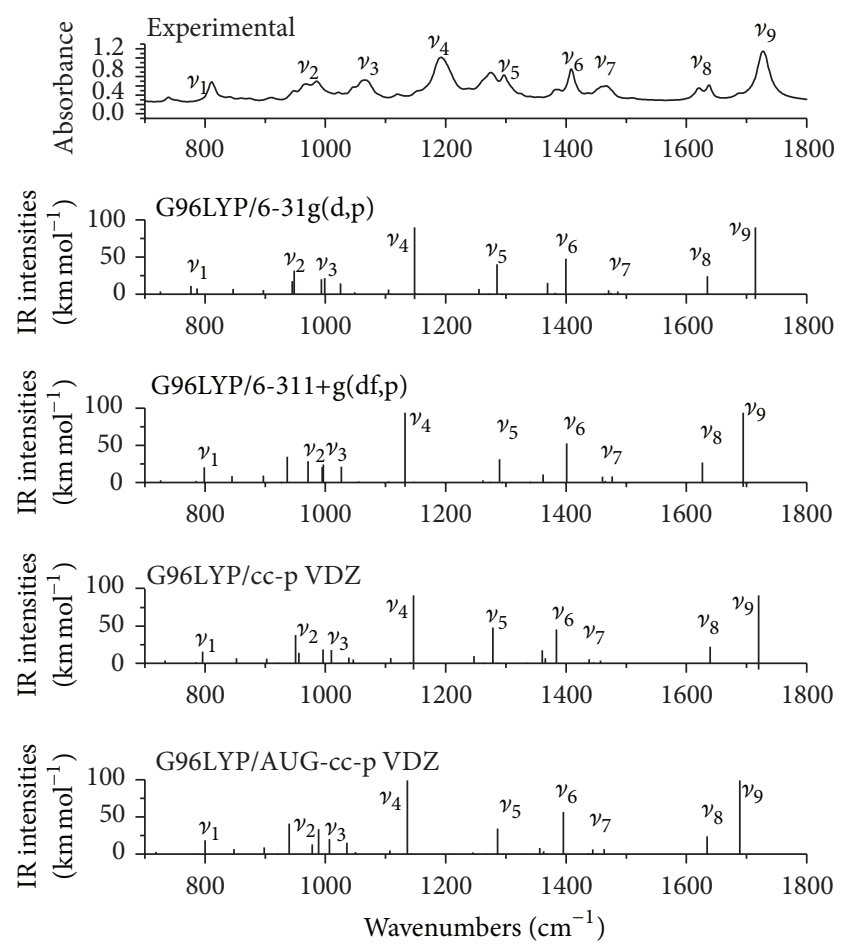

FIGURE 7: Comparison between the experimental infrared spectrum of butyl acrylate and the simulated spectra using different basis sets at the G96LYP functional. 

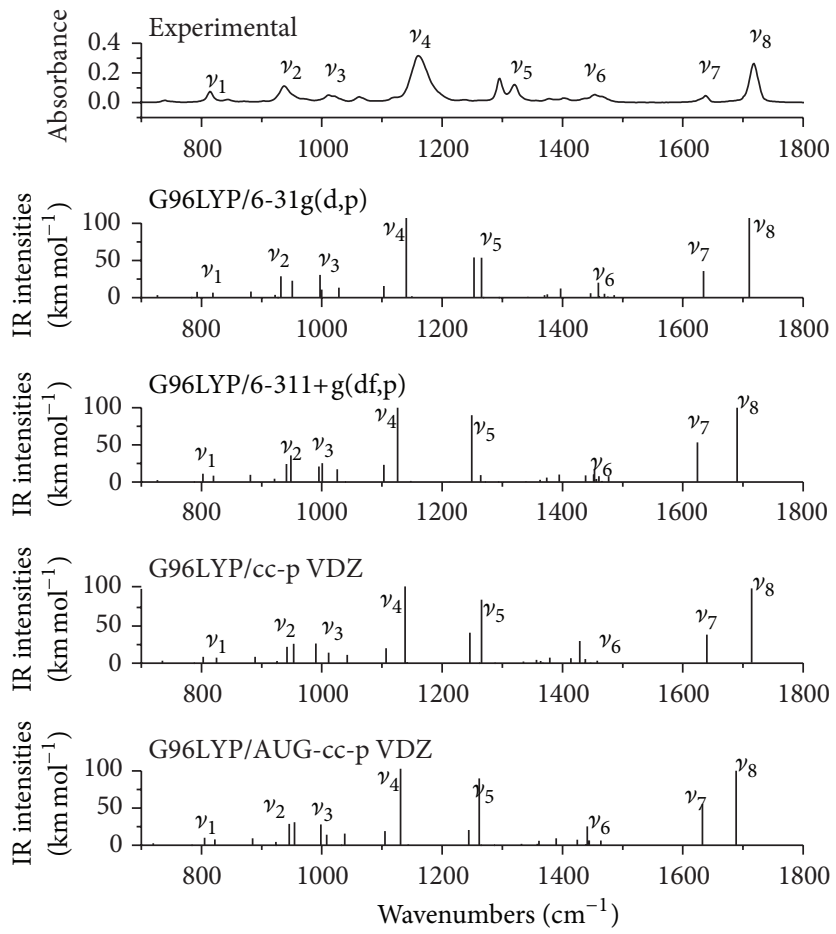

FIGURE 8: Comparison between the experimental infrared spectrum of butyl methacrylate and the simulated spectra using different basis sets at the G96LYP functional.
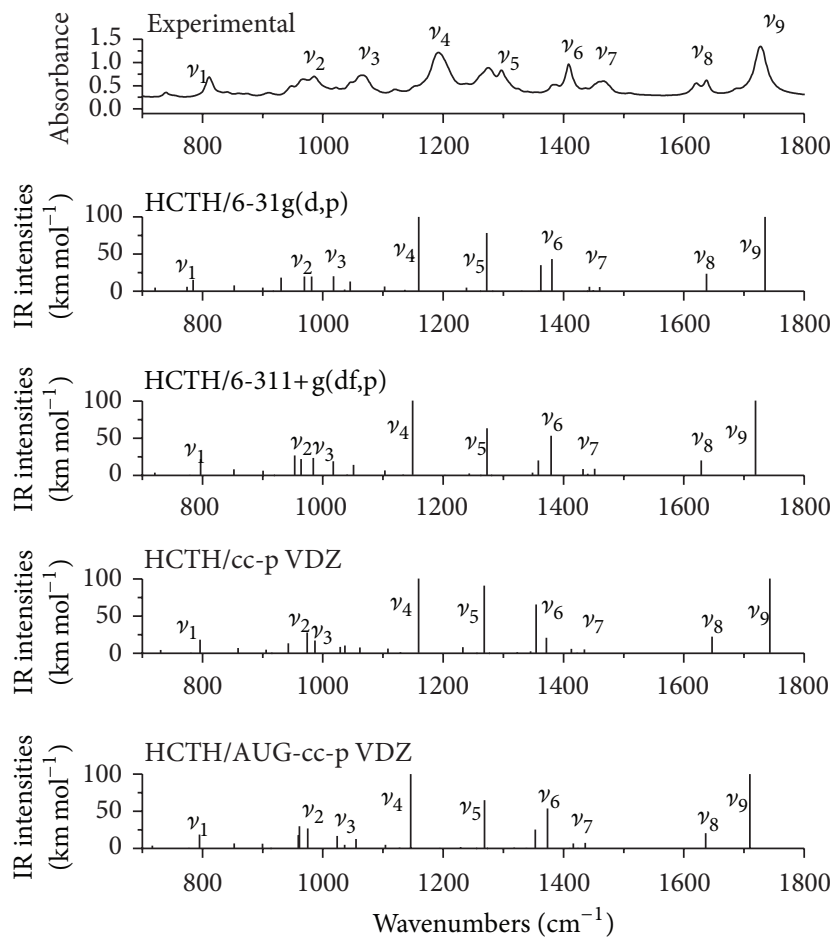

FIGURE 9: Comparison between the experimental infrared spectrum of butyl acrylate and the simulated spectra using different basis sets at the HCTH functional. 

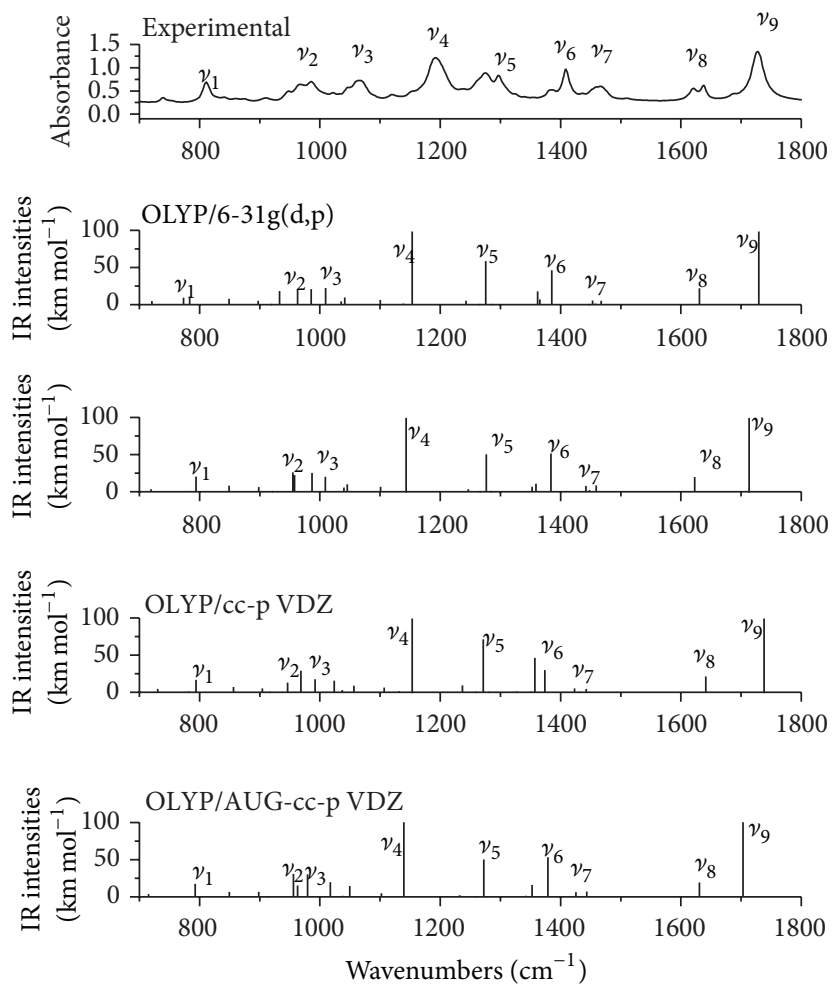

FIGURE 10: Comparison between the experimental infrared spectrum of butyl acrylate and the simulated spectra using different basis sets at the OLYP functional.
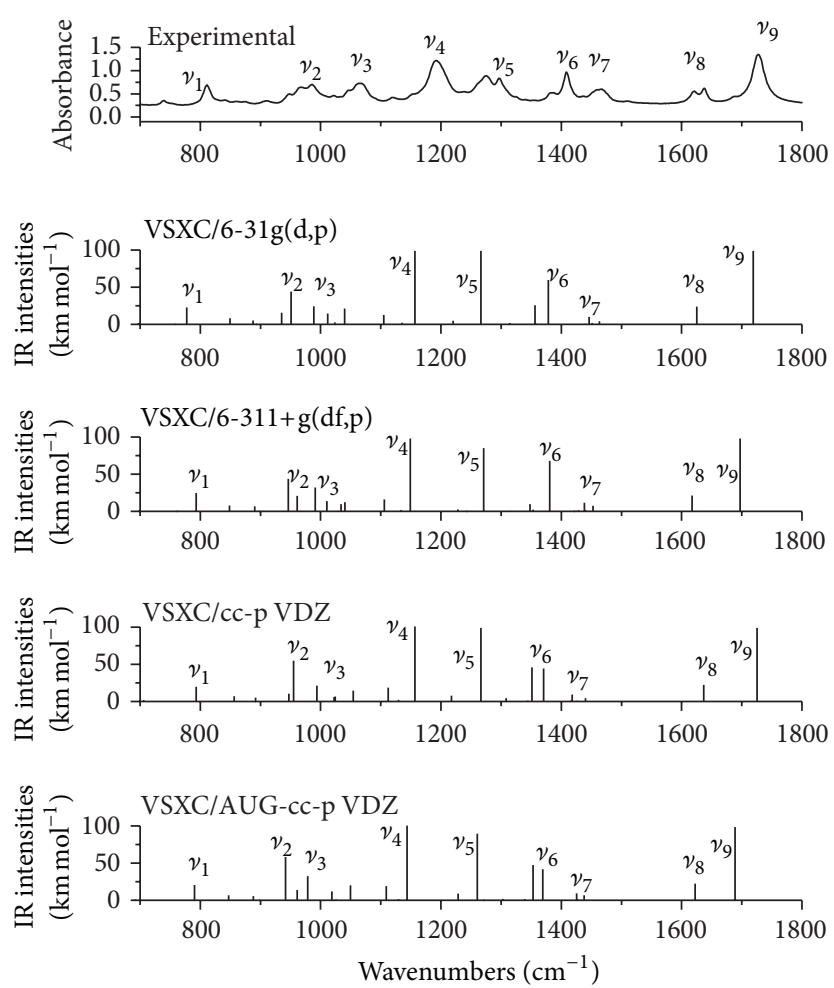

FIGURE 11: Comparison between the experimental infrared spectrum of butyl acrylate and the simulated spectra using different basis sets at the VSXC functional. 

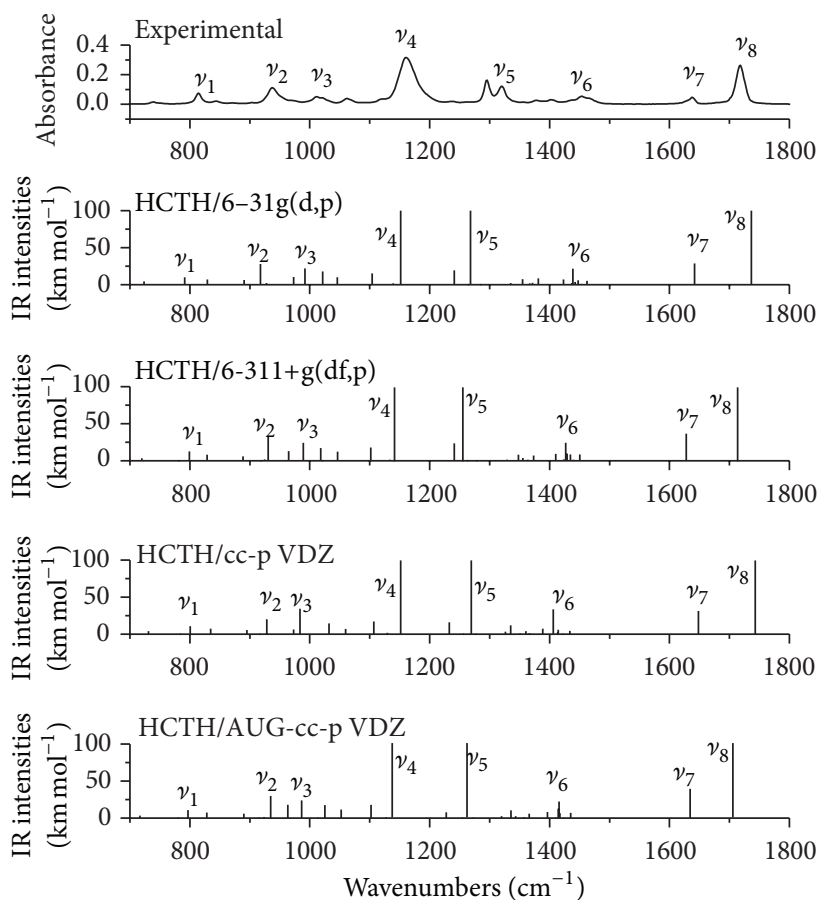

FIGURE 12: Comparison between the experimental infrared spectrum of butyl methacrylate and the simulated spectra using different basis sets at the HCTH functional.

OLYP, and VSXC functionals. The 6-31G(d,p) and cc-p VDZ basis sets give the best results among the four basis sets used for HCTH and VSXC functionals. The OLYP gives the worst values with Dunning's basis sets. The calculated frequencies for these three functionals show a good reproduction of the experimental spectrum in region 1200 to $1800 \mathrm{~cm}^{-1}$. In the region 700 to $1200 \mathrm{~cm}^{-1}$, the calculated frequencies less estimate the experimental bands. Nevertheless, this deficiency is in an acceptable range of $20 \mathrm{~cm}^{-1}$. The decrease of accuracy of these functionals is given in the order of increasing of $\% \Delta_{\text {vib }_{i}}$ as follows: HCTC6-31g(d,p) $(1.12 \%)<$ HCTC cc-pVDZ $(1.24 \%)<$ HCTC $6-311+\mathrm{g}(\mathrm{df}, \mathrm{p})(1.34 \%)<\operatorname{OLYP} 6-31 \mathrm{lg}(\mathrm{d}, \mathrm{p})$ $(1.46 \%)<$ HCTC aug-cc-pVDZ(1.6\%) < OLYP 6-311+g(df,p) $(1.67 \%)<$ VSXC cc-pVDZ $(1.76 \%)<\operatorname{VSXC6}-3 \lg (\mathrm{d}, \mathrm{p})(1.78 \%)$ $<$ OLYP cc-pVDZ $(2.35 \%)<$ VSXC 6-311+g(df,p) $(2.75 \%)<$ OLYP aug-cc-pVDZ (3.01\%) < VSXC aug-cc-pVDZ (3.96\%).

For BuMA molecule, the Pople's basis sets give the best results through the HCTH, OLYP, and O3LYP functionals (Figures 12 to 16). The decrease of accuracy of these functionals is given in the order of increasing of $\% \Delta_{\mathrm{vib}_{i}}$ as follows: O3LYP 6-31g(d,p) $(1.07 \%)<$ O3LYP 6-311+g(df,p) $(1.22 \%)$ $<$ O3LYP aug-cc-pVDZ (1.35\%) < OLYP6-31g(d,p) (1.44\%) $<\operatorname{HCTC6-31g(d,p)~(1.47\% )~<~O3LYP~cc-pVDZ~}(1.54 \%)<$ HCTC 6-311+g(df,p) $(1.8 \%)<$ OLYP 6-311+g(df,p) $(1.93 \%)<$ OLYP cc-pVDZ $(1.95 \%)<$ HCTC aug-cc-pVDZ $(2.06 \%)<$ HCTC cc-pVDZ (2.12\%) < OLYP aug-cc-pVDZ (2.14\%).

4.3. Good Correlated Spectra. The calculated and scaled frequencies at the O3LYP functional show a good correlations with the experimental infrared spectrum of the BuA molecule with Pople's basis sets see Figure 15. The majority of the calculated frequencies at O3LYP functional give an overestimation of the experimental bands. The frequencies go down about $15 \mathrm{~cm}^{-1}$ when the $6-311+\mathrm{G}(\mathrm{fd}, \mathrm{p})$ and augcc-p DVZ basis sets are used; however, they reproduce well the $v_{2}$ and $v_{3}$ bands. Regardless of inexactitudes related to the gas phase simulation environment, the O3LYP/6-31G(f,p) and O3LYP/6-311+G(df,p) levels of theory represent the best results for reproducing the infrared spectrum. The calculated $\% \Delta_{\text {vib }_{i}}$ is of about $0.5 \%$.

The percentage of square errors calculated for the scaled frequencies of BuMA revealed that the use of VSXC with Pople's basis sets gives the best correlations with the experimental spectrum (Figure 16) within all functionals used. The $\% \Delta_{\text {vib }_{i}}$ calculated are VSXC 6-31g(d,p) $(0.71 \%)<$ VSXC 6$311+\mathrm{g}(\mathrm{df}, \mathrm{p})(0.97 \%)$.

\section{Conclusion}

We have tested through this work the applicability and the capability of the scaling factors proposed in the literature to reproduce the experimental infrared spectra for two medium-sized molecules composed of about twenty-four atoms, ten of them are heavy. The B972 and B98 are not recommended for frequency calculation as they show a big deviation from the observed bands. The HCTH and OLYP functionals give acceptable results especially when we consider the environmental approximation between the gas phase simulation and the infrared spectra recorded in the liquid state. The O3LYP/6-31G(f,p) and O3LYP/6$311+\mathrm{G}(\mathrm{df}, \mathrm{p})$ levels of theory represent the best choice to study the infrared spectrum of butyl acrylate molecule. In the 

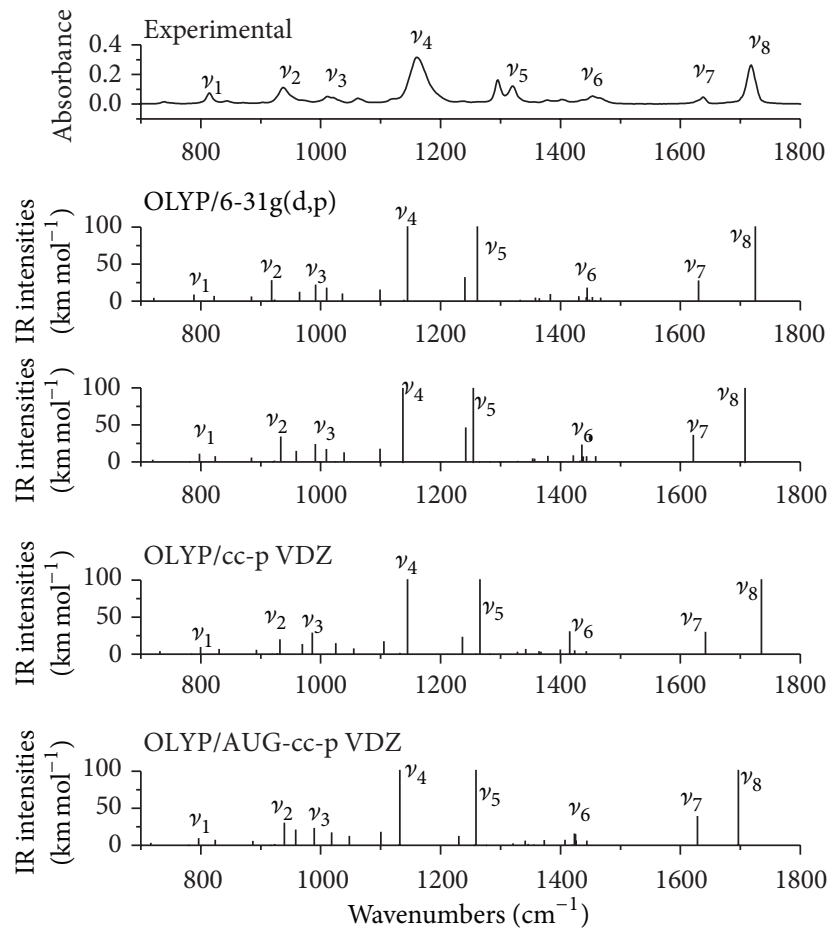

FIGURE 13: Comparison between the experimental infrared spectrum of butyl methacrylate and the simulated spectra using different basis sets at the OLYP functional.
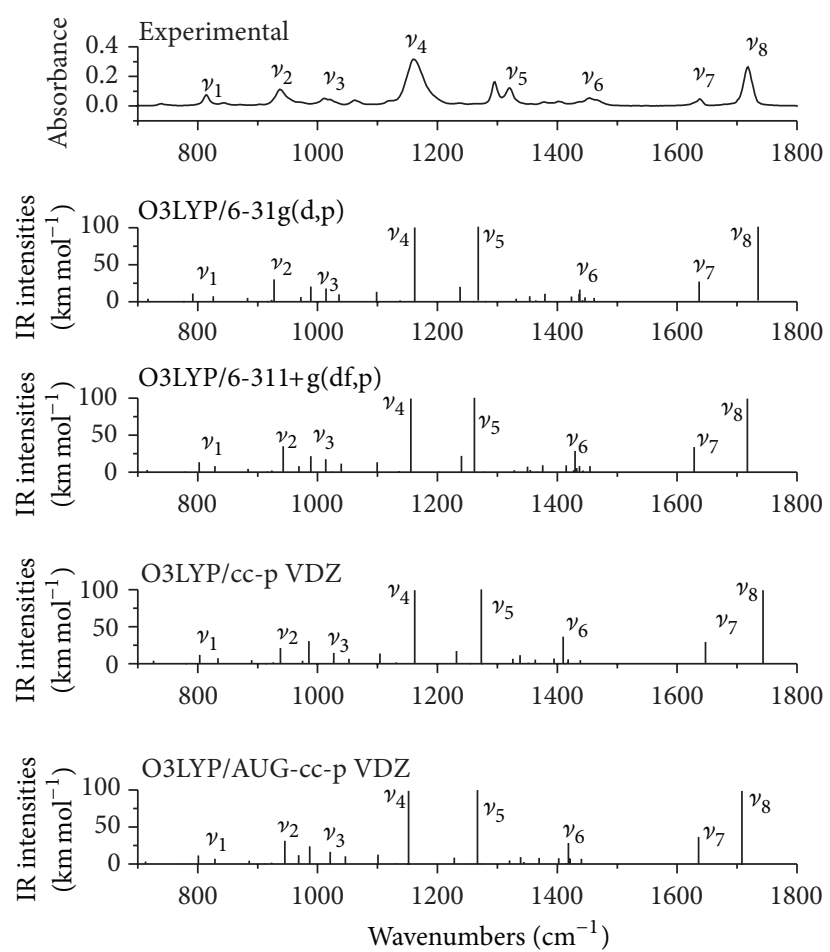

FIGURE 14: Comparison between the experimental infrared spectrum of butyl methacrylate and the simulated spectra using different basis sets at the O3LYP functional. 

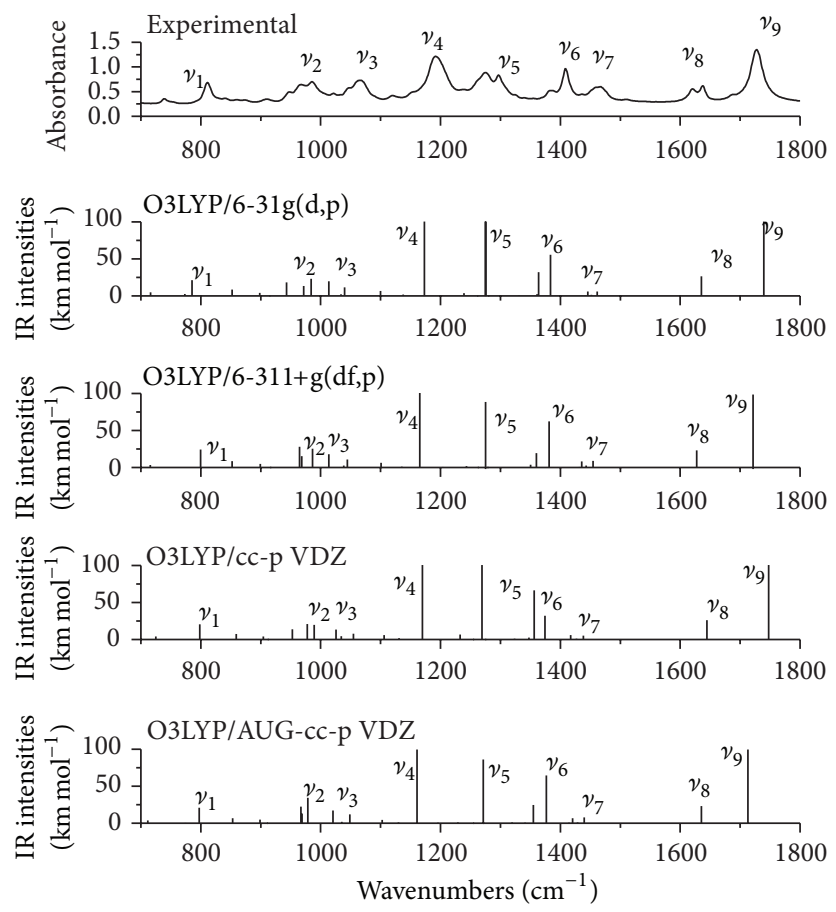

Figure 15: Comparison between the experimental infrared spectrum of butyl acrylate and the simulated spectra using different basis sets at the O3LYP functional.
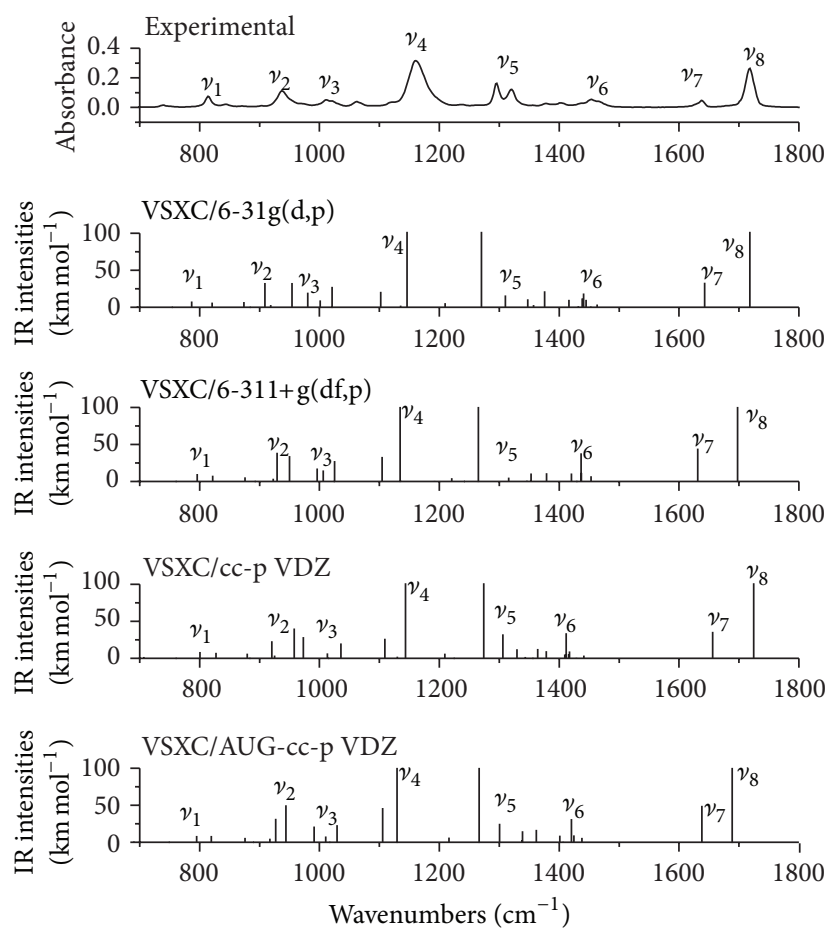

FIGURE 16: Comparison between the experimental infrared spectrum of butyl methacrylate and the simulated spectra using different basis sets at the VSXC functional. 
case of butyl methacrylate, VSXC/6-31g(d,p) shows the best results. Nevertheless, the hybrid DFT functional O3LYP is a good competitor for reproducing the infrared spectrum of the latter molecule. The G96LYP contains the Gill96 exchange functional, which has a rather simple form with only one parameter; thus, frequencies' scaling factors closest to 1 would be probably more concise for large molecules regarding time consuming.

The discrepancies between the obtained spectra within all the functionals used are probably due to the quality of the exchange correlation equations used. The method used to obtain the parameters of the functionals. The set of molecules used for parameterization and the fraction of HF exchange incorporated within the hybrid functionals play an important role to improve their quality.

\section{References}

[1] N. C. Handy and A. J. Cohen, "Left-right correlation energy," Molecular Physics, vol. 99, no. 5, pp. 403-412, 2001.

[2] W.-M. Hoe, A. J. Cohen, and N. C. Handy, "Assessment of a new local exchange functional OPTX," Chemical Physics Letters, vol. 341, no. 3-4, pp. 319-328, 2001.

[3] A. Virdi, V. P. Gupta, and A. Sharma, "Ab initio studies on conformation, vibrational and electronic spectra of methyl methacrylate," Journal of Molecular Structure: THEOCHEM, vol. 634, no. 5, pp. 53-65, 2003.

[4] T. Tsuji, H. Ito, H. Takeuchi, and S. Konaka, "Molecular structure and conformation of methyl methacrylate determined by gas electron diffraction," Journal of Molecular Structure, vol. 475, no. 1, pp. 55-63, 1999.

[5] B. L. Baker, M. Orgill, N. L. Owen et al., "The molecular conformation of methyl methacrylate-an infrared and ab initio study," Journal of Molecular Structure, vol. 356, no. 2, pp. 95-104, 1995.

[6] J. Lapić, A. Višnjevac, M. Cetina, S. Djaković, V. Vrček, and V. Rapić, "Experimental and DFT study of cyclodehydration and acetylation of ferrocenyl diols," The Journal of Molecular Structure, vol. 1019, no. 2, pp. 7-15, 2012.

[7] E. Gornicka, J. E. Rode, E. D. Raczynska, B. Dasiewicz, and J. Dz. Dobrowolski, "Experimental (FT-IR and Raman) and theoretical (DFT) studies on the vibrational dynamics in cytisine," Vibrational Spectroscopy, vol. 36, no. 1, pp. 105-115, 2004.

[8] T. Egawa, S. Maekawa, H. F. Takeuchi, and S. Konaka, "Molecular structure and conformation of methyl acrylate. A gas electron diffraction study augmented by ab initio calculation and rotational constants," Journal of Molecular Structure, vol. 352, no. 1, pp. 193-201, 1995.

[9] P. Pulay, G. Fogarasi, F. Pang, and J. E. Boggs, "Systematic ab initio gradient calculation of molecular geometries, force constants, and dipole moment derivatives," Journal of the American Chemical Society, vol. 101, no. 10, pp. 2550-2560, 1979.

[10] J. Baker, A. A. Jarzecki, and P. Pulay, "Direct scaling of primitive valence force constants: an alternative approach to scaled quantum mechanical force fields," Journal of Physical Chemistry A, vol. 102, no. 8, pp. 1412-1424, 1998.

[11] Y. Tantirungrotechai, K. Phanasant, S. Roddecha, P. Surawatanawong, V. Sutthikhum, and J. Limtrakul, "Scaling factors for vibrational frequencies and zero-point vibrational energies of some recently developed exchange-correlation functionals,"
Journal of Molecular Structure: THEOCHEM, vol. 760, no. 1-3, pp. 189-192, 2006.

[12] A. D. Becke, "Density-functional thermochemistry. V. Systematic optimization of exchange-correlation functionals," Journal of Chemical Physics, vol. 107, no. 20, pp. 8554-8560, 1997.

[13] P. J. Wilson, T. J. Bradley, and D. J. Tozer, "Hybrid exchangecorrelation functional determined from thermochemical data and ab initio potentials," Journal of Chemical Physics, vol. 115, no. 20, pp. 9233-9242, 2001.

[14] H. L. Schmider and A. D. Becke, "Optimized density functionals from the extended G2 test set," Journal of Chemical Physics, vol. 108, no. 23, pp. 9624-9631, 1998.

[15] P. M. W. Gill, "A new gradient-corrected exchange functional," Molecular Physics, vol. 89, no. 2, pp. 433-445, 1996.

[16] F. A. Hamprecht, A. J. Cohen, D. J. Tozer, and N. C. Handy, "Development and assessment of new exchange-correlation functionals," Journal of Chemical Physics, vol. 109, no. 15, pp. 6264-6271, 1998.

[17] T. van Voorhis and G. E. Scuseria, "A novel form for the exchange-correlation energy functional," Journal of Chemical Physics, vol. 109, no. 2, pp. 400-410, 1998.

[18] M. J. Frisch, G. W. Trucks, H. B. Schlegel et al., Gaussian 03, Revision B.01, Gaussian, Inc., Pittsburgh, Pa, USA, 2003.

[19] J. Jaramillo and G. E. Scuseria, "Performance of a kinetic energy density-dependent functional (VSXC) for predicting vibrational frequencies," Chemical Physics Letters, vol. 312, no. 2-4, pp. 269-276, 1999.

[20] G. Jovanovski, A. Ćahil, O. Grupče, and L. Pejov, "Vibrational analysis of thiosaccharin and thiosaccharinate anion. A gradient-corrected density functional and experimental study," Journal of Molecular Structure, vol. 784, no. 1-3, pp. 7-17, 2006.

[21] H. Raissi, A. Nowroozi, M. Roozbeh, and F. Farzad, "Molecular structure and vibrational assignment of (trifluoroacetyl) acetone: a density functional study," Journal of Molecular Structure, vol. 787, pp. 148-162, 2005.

[22] W. Kohn and L. J. Sham, "Self-consistent equations including exchange and correlation effects," Physical Review, vol. 140, no. 4A, pp. A1133-A1138, 1965.

[23] W. J. Hehre, K. Ditchfield, and J. A. Pople, "Self-consistent molecular orbital methods. XII. Further extensions of gaussiantype basis sets for use in molecular orbital studies of organic molecules," The Journal of Chemical Physics, vol. 56, no. 5, pp. 2257-2261, 1972.

[24] P. C. Hariharan and J. A. Pople, "The influence of polarization functions on molecular orbital hydrogenation energies," Theoretica Chimica Acta, vol. 28, no. 3, pp. 213-222, 1973.

[25] R. Krishnan, J. S. Binkley, R. Seeger, and J. A. Pople, "Selfconsistent molecular orbital methods. XX. A basis set for correlated wave functions," The Journal of Chemical Physics, vol. 72, no. 1, pp. 650-654, 1980.

[26] T. H. Dunning Jr., "Gaussian basis sets for use in correlated molecular calculations. I. The atoms boron through neon and hydrogen," The Journal of Chemical Physics, vol. 90, no. 2, pp. 1007-1023, 1989. 

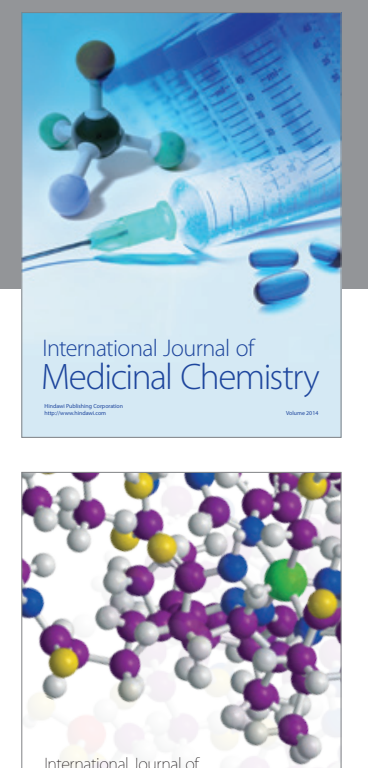

\section{Carbohydrate} Chemistry

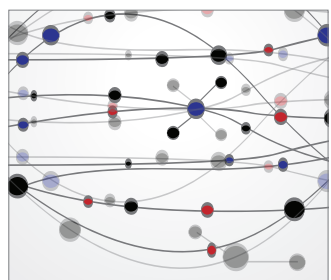

The Scientific World Journal
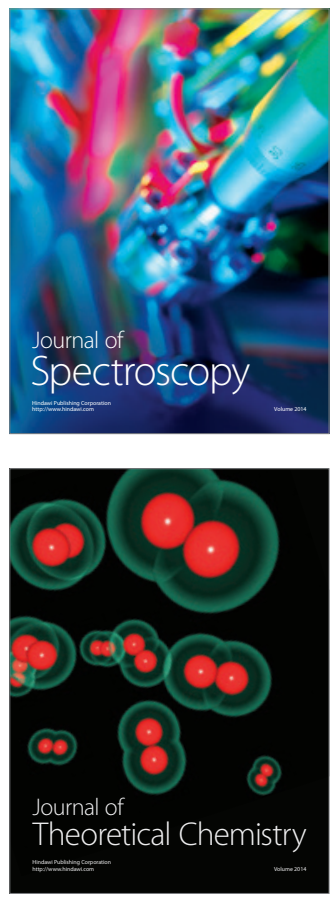
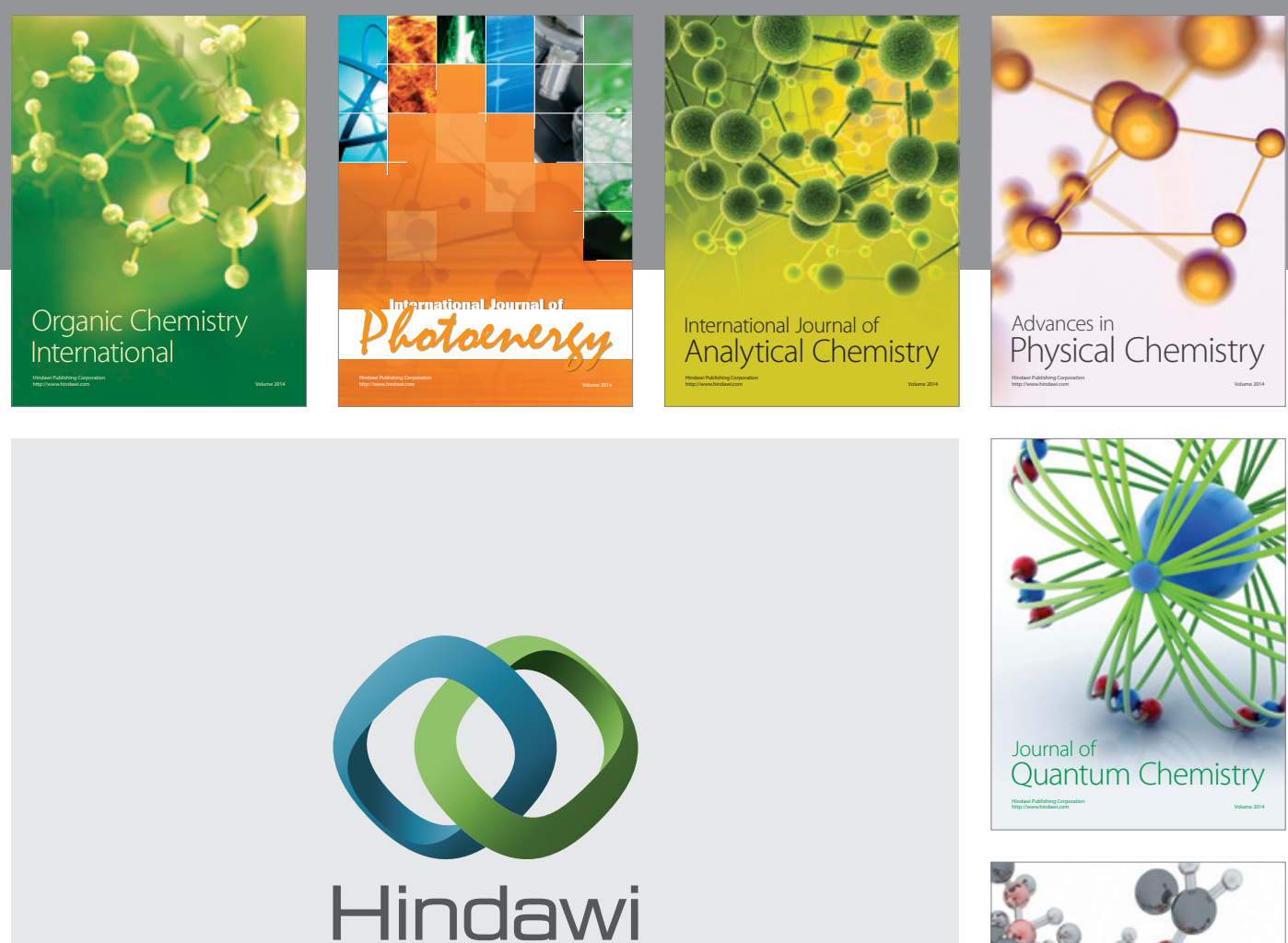

Submit your manuscripts at

http://www.hindawi.com

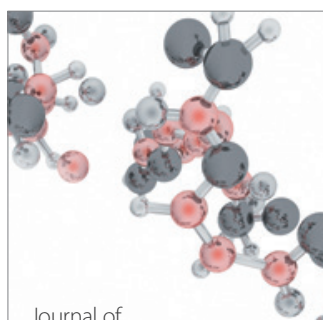

Analytical Methods

in Chemistry

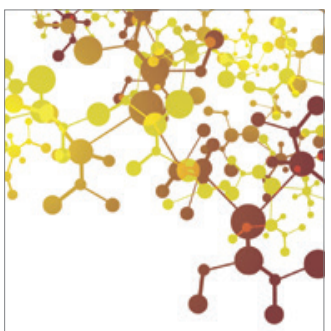

Journal of

Applied Chemistry

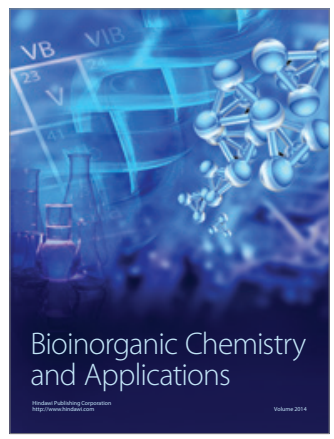

Inorganic Chemistry
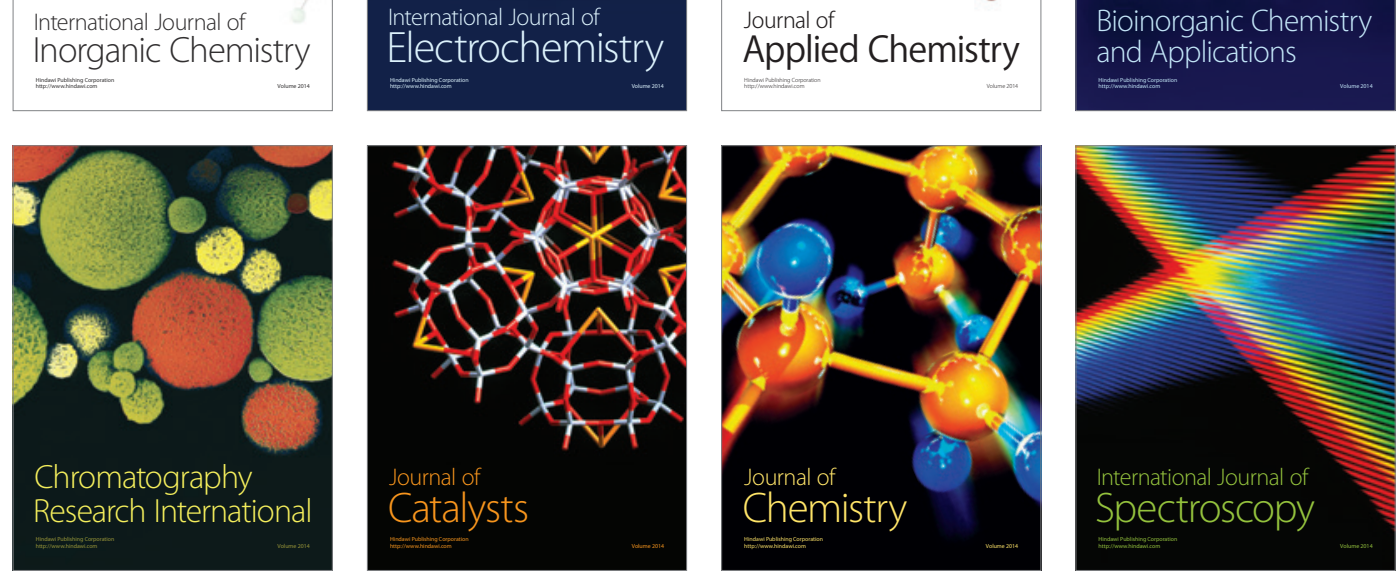